\title{
Algunas consideraciones sobre el factor edad en relación con la enseñanza de las lenguas extranjeras en la escuela
}

\author{
Julio Roca de Larios \\ Rosa María MANchón RuIZ \\ Universidad de Murcia
}

Recibido: 27 enero 2005 / Versión aceptada: 27 junio 2005

ISSN: $1697-7467$

\begin{abstract}
RESUMEN: El propósito del presente artículo es analizar de forma crítica algunas de las ideas derivadas de la investigación sobre el factor edad que muestran cierta relevancia para una fundamentación de la enseñanza de lenguas extranjeras en primaria. Se presentan, en primer lugar, algunos de los estudios en los que se ha hallado evidencia empírica tanto a favor como en contra de que los niños son mejores aprendices de lenguas que los adultos y, a continuación, se sugiere un conjunto de conclusiones de cara a la enseñanza de las lenguas extranjeras en la escuela.

Palabras clave: factor edad, aprendizaje de lenguas, contexto natural, contexto formal.

ABSTRACT: The purpose of this paper is to offer a critical analysis of the empirical research on age related differences which may be relevant to justify the introduction of foreign languages in the primary school. First, we discuss some of the empirical evidence both for and against the idea that children are better second language learners than adults. Finally, a number of conclusions related to the teaching of foreign languages at primary school level are suggested.
\end{abstract}

Key words: age factor, language learning, natural context, formal context.

\section{INTRODUCCIÓN}

La supuesta capacidad de los niños para aprender idiomas con mayor facilidad y perfección que los adultos es una creencia muy extendida que se halla incluso documentada. Así, por ejemplo, en un estudio llevado a cabo en Cataluña con padres de alumnos que habían comenzado a estudiar inglés antes de los ocho años se halló que el 92\% de los mismos creía en las ventajas de los niños respecto a los mayores de cara al aprendizaje de segundas lenguas (Torras, Tragant y García, 1997). En el País Vasco se ha observado igualmente que los padres, profesores y tutores piensan que el aprendizaje del inglés puede tener efectos positivos no sólo sobre el aprendizaje del euskera y del castellano, sino también sobre el desarrollo cognitivo de los alumnos y su rendimiento en otras asignaturas (Cenoz, 2003a). Estas creencias, que hunden sus raíces en la posible existencia de un periodo evolutivo, o periodo crítico, que duraría hasta la pubertad y en el que sería posible la adquisición de una lengua añadida a niveles semejantes a los alcanzados por los hablantes nativos, parten de la constatación de 
que, mientras que para los niños normales es casi un derecho de nacimiento alcanzar la competencia total en la lengua que empiezan a aprender, en el caso de los adultos se suele producir una enorme variabilidad en el nivel de competencia alcanzado en la segunda lengua (L2), siendo este nivel, además, muy raras veces comparable al de los hablantes nativos.

En la investigación sobre la adquisición de la lengua materna (L1), la existencia de un periodo crítico es un hecho generalmente admitido (véase, sin embargo, Singleton, 2003). En este ámbito hay todo un conjunto de evidencias derivadas de estudios con niños salvajes, con niños que han sufrido dificultades auditivas severas (Ruben, 1997) y con aprendices de lenguajes de signos (Morford y Mayberry, 2000) que han llevado a muchos investigadores a concluir que, cuando el contacto con la L1 se aplaza de forma sustantiva, la competencia que se puede alcanzar es irregular y limitada, sin que ningún tipo de experiencia posterior pueda compensar esa privación inicial (Long, 1990). Por otra parte, hay toda una serie de fenómenos relativos a la existencia de determinadas secuencias de adquisición, como, por ejemplo, la aparición de ciertas estructuras sintácticas de transición o la presencia de determinados tipos de errores impenetrables a la influencia del medio ambiente, que parecen sugerir que el aprendizaje de la lengua materna sólo se puede entender si se asume que hay "determinados aspectos significativos del desarrollo lingüístico que están dictados por nuestra biología" (Gleitman y Newport, 1995: 10).

La controversia surge, no obstante, cuando la Hipótesis del Periodo Crítico se quiere hacer extensiva a la adquisición de la L2, pues en este campo no sólo se carece de una explicación que cuente con un mínimo de consenso entre los investigadores, sino que la misma existencia del fenómeno es minimizada por algunos e incluso negada por otros (De Keyser, 2000). El tema, sin embargo, tiene importantes implicaciones teóricas y prácticas. Desde un punto de vista teórico, el debate sobre la existencia del periodo crítico encierra algunas de las preguntas más relevantes sobre la adquisición de una segunda lengua: si su aprendizaje se rige por factores ambientales o por un programa interno del aprendiz, si las representaciones mentales de la L1 y de la L2 son construidas por los hablantes bilingües de forma independiente o en común, o si la transferencia es un proceso legítimo en el aprendizaje de lenguas o un síntoma no deseado de la separación de las mismas (Bialystok y Hakuta, 1999). En este sentido, la existencia de un período crítico sería compatible con la idea de que los humanos se hallan equipados con mecanismos internos para la adquisición del lenguaje y sugeriría la existencia de secuencias de desarrollo que estarían genéticamente determinadas (Skehan, 1998).

Desde el punto de vista práctico, la existencia de un periodo crítico iría necesariamente asociada a una serie de limitaciones relativas al nivel de competencia última que se podría alcanzar en el aprendizaje de una L2 y debería conducir, por tanto, a un cierto realismo con relación a los objetivos que cabría plantearse desde el punto de vista educativo (Skehan, 1998). Si se demostrase que el periodo crítico es esencial para alcanzar un nivel de competencia lingüística semejante al que muestran los nativos, el aprendizaje tardío de la lengua tendría necesariamente que basarse en objetivos modestos y, además, afectaría al tipo de metodología utilizada en el aula. En efecto, la falta de acceso a la gramática universal (GU) por parte del aprendiz que empezase su contacto con la L2 una vez que la "ventana de oportunidad" (Schachter, 1996) se hubiese cerrado, le obligaría a recurrir a habilidades y recursos cognitivos de carácter general para habérselas con la tarea de aprendizaje y, como resultado, las estrategias de enseñanza habrían de estar en consonancia con dichas habilidades y recursos. 
Por otra parte, la existencia de un periodo crítico proporcionaría argumentos bastantes razonables para la introducción temprana del estudio de lenguas extranjeras en la escuela, a fin de que los niños pudiesen obtener el máximo provecho de las especiales capacidades para aprender lenguas que se darían en ese periodo de su vida. El caso del sistema educativo español, donde las lenguas extranjeras se introdujeron inicialmente en la EGB a los 11 años, para ser adelantadas posteriormente a los ocho, después a los $6 \mathrm{y}$, posiblemente, a la etapa de Infantil en el próximo futuro, es un claro ejemplo de la tendencia general a empezar la enseñanza de las lenguas extranjeras "a niveles cada vez más bajos, en la creencia de que esta política podrá dar lugar a todo un conjunto de ciudadanos capaces de hablar la L2 de forma fluida" (Scovel, 2000: 213-214).

En las páginas que siguen se analizarán algunas de las ideas derivadas de la investigación sobre el factor edad que presentan cierta relevancia para una fundamentación de la enseñanza de lenguas extranjeras en la escuela. No se pretende, por tanto, hacer una revisión exhaustiva de la literatura sobre el tema ni dar cuenta de las distintas explicaciones teóricas propuestas (para ello pueden consultarse, por ejemplo, las revisiones de Birdsong, 1999; Harley y Wang, 1997; Singleton, 2001; o Hyltenstam y Abrahamsson, 2003). En su lugar, se presentarán, en primer lugar, algunos de los estudios en los que se ha hallado evidencia empírica tanto a favor como en contra de la idea de que los niños son mejores aprendices que los adultos y, a continuación, se cerrará el trabajo con las conclusiones que de dicha discusión se puedan extraer de cara a la enseñanza del inglés en la escuela.

\section{EVIDENCIA A FAVOR DE QUE LOS NIÑOS SON MEJORES APRENDICES DE LE QUE LOS ADULTOS}

Dejando a un lado la investigación en la que se ha documentado la existencia de diferencias debidas a la edad por medio de técnicas de exploración cerebral ${ }^{1}$, son sobre todo los análisis de las producciones de los aprendices de L2 en contextos naturales los que han servido de base para apoyar la posición que Singleton (1989) caracteriza como la versión fuerte de la Hipótesis del Periodo Crítico: los aprendices de menor edad son más eficaces y tienen más éxito en el aprendizaje que los mayores, y la pubertad marca el comienzo del declive en la capacidad para adquirir la segunda lengua. A continuación resumiremos algunos de estos estudios.

${ }^{1}$ Kim y otros (1997), por ejemplo, utilizaron la técnica de la resonancia magnética y hallaron diferencias en el área de Broca entre bilingües tempranos y tardíos durante la realización de una tarea de generación de frases. Los primeros mostraban un solo centro de activación para la primera y la segunda lengua, mientras que los tardíos mostraban dos, uno para cada lengua, lo que indicaba, según los autores, la existencia de diferentes tipos de organización cerebral según la edad de inicio de aprendizaje de la LE. Weber-Fox y Neville (1999) también llevaron a cabo experimentos utilizando técnicas de exploración cerebral y hallaron, de forma consistente, diferencias entre niños y adultos con relación a los patrones de activación cerebral y la localización del procesamiento del lenguaje. También hallaron que algunas anomalías gramaticales estaban relacionadas con la edad, de forma que para algunas de ellas los 4 años constituirían el fin de un determinado periodo crítico, mientras que, para otras, el límite habría que situarlo a los 11 años de edad. 


\subsection{Estudios en contextos naturales}

En los estudios realizados en contextos naturales el procedimiento de investigación más utilizado ha consistido en analizar la producción lingüística -especialmente la pronunciación y la corrección morfosintáctica y, en menor medida, el léxico- de inmigrantes que han aprendido la L2 en un país de acogida y compararla con la producción de los nativos de ese mismo país. Con relación a la pronunciación, los resultados muestran de forma regular que, a largo plazo, los aprendices que llegan al país antes de los 6-7 años de edad suelen producir la L2 con menos acento extranjero que quienes llegan a edades posteriores (Harley y Wang, 1997; Flege, 1999). De hecho, aunque el tiempo de residencia en el país puede ser un factor más determinante que la edad de llegada al mismo durante los meses iniciales de estancia, es esta última variable -la edad de llegada- la que, a partir de los dos años de residencia, parece más importante a la hora de predecir el nivel de pronunciación. Thompson (1991), por ejemplo, halló que, entre un grupo de rusos que habían emigrado a los EEUU entre los 4 y los 44 años de edad, la edad de llegada al país de acogida era el mejor indicador de la corrección de su pronunciación en inglés. Más recientemente, dos estudios realizados por Flege y sus colegas, en los que participaron emigrantes italianos residentes en Canadá (Flege y otros, 1995) y coreanos en EEUU (Flege y otros, 1999), también mostraron que la calidad del acento al hablar una L2 mostraba una fuerte correlación con la edad de llegada de los participantes a los respectivos países de acogida: los que habían llegado siendo muy jóvenes tenían un acento menos marcado. Todo ello parece corroborar la conclusión a la que llegó Long (1990) en una revisión de estudios sobre el tema: que la L2 se puede hablar con poco acento si su aprendizaje en el medio natural empieza antes de los seis años de edad, pero que se suele hablar con acento si se empieza después de los doce, y con éxito variable si el comienzo tiene lugar entre los seis y los doce.

Por lo que respecta a los aspectos gramaticales de la L2, la investigación en contextos naturales arroja resultados semejantes a los anteriores. Patkowski (1980), por ejemplo, halló una alta correlación entre la edad de llegada de un grupo de inmigrantes a los EEUU y el nivel de corrección sintáctica que mostraban en unas entrevistas orales en inglés: quienes habían llegado antes de los quince años recibieron puntuaciones medias muy superiores a las que obtuvieron los que habían llegado después de esa edad. Resultados semejantes fueron documentados por Hyltenstam (1992), quien analizó las versiones oral y escrita de un cuento realizadas por un grupo de españoles y finlandeses aprendices de sueco como L2 y las comparó con las narraciones de un grupo de nativos. Los datos indicaron que apenas había coincidencia en la distribución de los errores cometidos por los nativos y los cometidos por los aprendices que habían adquirido el sueco después de los siete años, pero que sí la había en los errores cometidos por los nativos y por los aprendices que habían adquirido la L2 antes de los seis años de edad. Johnson y Newport (1989) hallaron, mediante juicios orales de gramaticalidad centrados en una amplia gama de estructuras morfosintácticas del inglés (276 ítems), que los emigrantes chinos y coreanos llegados a los EEUU antes de los siete años de edad tenían un rendimiento semejante al de los nativos, mientras que se producía un declive de carácter lineal cuando la edad de llegada se situaba entre los siete y los diecisiete años. A partir de esta edad, los participantes mostraban una gran variabilidad en cuanto a su competencia en inglés (aspecto que no se observó entre aquellos que habían llegado a una edad temprana), pero no se halló ningún tipo de efecto en razón de su edad. Johnson (1992) 
replicó el estudio con los mismos sujetos un año después en un formato escrito y halló que los resultados eran esencialmente los mismos, aunque las correlaciones entre la edad y las puntuaciones en la prueba fueron algo menores.

A pesar de que estos dos últimos estudios han atraído considerable atención en los últimos años y se han aducido como pruebas a favor del periodo crítico, no se han visto libres de críticas. La más importante se basa en la consideración de que el chino y el coreano -la L1 de los participantes- y el inglés -su L2- son muy distantes entre sí, lo que ha llevado a especular sobre el hecho de que los efectos supuestamente debidos a la edad bien pudieran deberse a diferencias tipológicas entre las lenguas. Todo ello, junto con otras consideraciones de tipo metodológico, llevó a DeKeyser (2000) a replicar el estudio de Johnson y Newport (1989) por medio de una versión modificada de la prueba de juicios de gramaticalidad empleada por éstos. Los participantes fueron un grupo de húngaros que llevaban un mínimo de 10 años residiendo en los EEUU, país al que, según los casos, habían llegado entre el primer año de vida y los 40 años de edad. Los resultados indicaron que se daba una correlación negativa entre las puntuaciones obtenidas y la edad de inicio del aprendizaje del inglés (antes o después de los 16 años), sin que otras variables tales como el tiempo de residencia en el país o los años de escolaridad resultasen significativas. Por otra parte, el estudio mostró que algunas estructuras gramaticales son más sensibles que otras a los efectos de la edad.

Finalmente, en un estudio centrado en el léxico, Mägiste (1987) analizó el rendimiento de un grupo de hablantes nativos de alemán de primaria y secundaria (que habían residido en Suecia durante diversos periodos de tiempo) en una tarea en la que tenían que poner nombre tanto en alemán como en sueco a dibujos de objetos y a números de dos dígitos. Con relación a los objetos, los resultados indicaron que los tiempos de respuesta en las dos lenguas por parte de los alumnos de primaria tendían a igualarse después de 4 años de residencia en Suecia, pero que había que esperar a 6 años para que esta igualación se produjese entre los participantes del grupo de secundaria. Por lo que respecta a los números, los tiempos de respuesta de ambos grupos en ambas lenguas coincidieron prácticamente en el mismo punto, es decir, al cabo de 3 o 4 años de residencia. Lo sorprendente respecto a este último resultado es que los aprendices del grupo elemental, a pesar de que esta tarea entrañaba más dificultad para ellos, alcanzaron el equilibrio entre las dos lenguas al mismo tiempo que los de secundaria. Ello llevó al autor a postular que su desventaja cognitiva general, respecto a los aprendices de más edad, quedaba compensada por su facilidad en la adquisición del léxico.

La conclusión que se deriva de todo este volumen de investigación es que la edad de inicio es un importante factor a considerar para explicar el dominio último de la L2 en contextos naturales. Sin embargo, autores como White y Genesse (1996) señalan que estos trabajos sólo muestran que el nivel de dominio último de la lengua que los aprendices tempranos alcanzan suele ser más alto que el alcanzado por los aprendices tardíos -un hecho que, a día de hoy, prácticamente nadie niega-, pero que de ello no se puede concluir que los aprendices de una L2, aunque inicien su aprendizaje a edades muy tempranas, llegan a alcanzar niveles de dominio semejantes a los nativos monolingües. De hecho, hay investigaciones que señalan que éste no es enteramente el caso, al menos en lo que respecta al empleo de determinadas estructuras gramaticales o a la calidad del vocabulario (véase Hyltestam y Abrahamsson, 2003). Incluso en el dominio de la pronunciación, Piske, Mackay y Flege (2001), en una revisión de estudios sobre el tema, concluyeron que, si bien la investigación 
ha mostrado que los aprendices tempranos pueden llegar a hablar la segunda lengua con un acento extranjero menos marcado que los aprendices tardíos, ningún estudio ha producido hasta la fecha evidencia convincente a favor del supuesto de que la producción oral de la L2 está libre de acento si se aprende antes de los 6 años. Antes bien, parece que existen otros factores, además de la edad de inicio del aprendizaje, que también tienen su influencia en la aparición de acento: la edad cronológica, el tiempo de residencia en el país en el que se habla la segunda lengua o la frecuencia de uso de la L1 y la L2. De ahí que Singleton (2003) sugiriera que, a la hora de estudiar los efectos de la edad en el aprendizaje de la L2, la comparación más apropiada no es tanto entre aprendices tardíos y hablantes monolingües como entre aprendices tardíos y tempranos ${ }^{2}$.

\section{Evidencia EN LA QUE SE CUESTIONA LA SUPERIORIDAD DE LOS NIÑoS}

Frente a la perspectiva anterior, hay otro grupo de estudios en los que se defiende la hipótesis de que los aprendices de más edad son comparables a los más jóvenes e incluso, a veces, más eficaces en el aprendizaje de la L2. Generalmente, esta posición ha venido avalada por estudios en los que (i) se ha analizado el nivel de competencia alcanzado por algunas personas que empezaron a estudiar la lengua extranjera a una edad adulta, o (ii) se ha evaluado la introducción de programas de enseñanza de lenguas extranjeras en la escuela ${ }^{3}$.

\subsection{El dominio de la $\mathrm{L} 2$ por parte de algunos adultos}

Se sabe que hay aprendices con un contacto tardío con la L2 que, sin embargo, son capaces de lograr niveles de competencia equiparables a los niveles de los nativos. Este hecho, poco conocido en parte por la tendencia a presentar el rendimiento de los adultos desde una perspectiva grupal (Marinova-Todd y otros, 2000), ha empezado a ser documentado de forma cada vez más explícita. En el área de la morfosintaxis, Birdsong (1992), por ejemplo, halló que el rendimiento global de un grupo de aprendices de francés residentes en Francia una media de tres años (y que habían empezado su aprendizaje entre los 11 y los 28

${ }^{2}$ Tomarse en serio esta observación llevaría a cuestionar los presupuestos teóricos desde los que se ha construido la noción de "nivel de dominio último". Cook (1992) ha sugerido que la utilización de este concepto en L2 no es correcta porque la multicompetencia de los bilingües es diferente del conocimiento lingüístico propio de los hablantes monolingües y que, por tanto, la competencia de los primeros no ha de ser juzgada con las normas propias de los segundos. Se sabe que un alto nivel de multicompetencia no es un resultado automático de la exposición a la segunda lengua, como lo demuestran, por ejemplo, los procesos de debilitamiento de la lengua materna sufridos por los niños de lenguas minoritarias o las influencias del sistema fonológico de la L2 sobre el de la L1 entre emigrantes adultos. Por otra parte, también se ha documentado que la multicompetencia aporta habilidades de tipo metalingüístico de naturaleza diferente a las que poseen los hablantes monolingües y que, por ello, parece razonable cuestionar los estudios basados en comparaciones de juicios de gramaticalidad de unos y otros.

${ }^{3}$ Dentro de esta perspectiva, hay un tercer grupo de estudios -que no trataremos aquí- relativo al aprendizaje de la L2 en situaciones de inmersión. Nikolov (2000) sugiere que estos estudios son relevantes para la enseñanza de las lenguas extranjeras porque sus datos, al fin y al cabo, han sido obtenidos en un contexto formal. Sin embargo, señala la autora que las técnicas de evaluación y de diseño utilizadas arrojan dudas sobre sus conclusiones y que, por lo tanto, es imposible tomar partido por un tipo de inmersión temprana o tardía. 
años de edad) era inferior al rendimiento de los nativos en una serie de juicios de gramaticalidad. Sin embargo, el hecho de que algunos aprendices adultos mostraran un nivel superior al de alguno de los nativos motivó que el autor cuestionara la vieja creencia de que las habilidades lingüísticas de los adultos tienden a fosilizarse en algún estadio de desarrollo (véase Selinker, 1972). White y Genesee (1996) estudiaron en Montreal la adquisición del inglés por parte de un grupo de hablantes de francés y hallaron que algunos de los que mostraban un rendimiento semejante al de los nativos en las pruebas propuestas -que consistían en reformulaciones de estructuras- habían empezado su aprendizaje después de los 12 años. Como último ejemplo en este campo citaremos a Cranshaw (1997, en Birdsong, 1999) que, también en Canadá, investigó la adquisición de los tiempos verbales en inglés por parte de un grupo de francófonos adultos. Después de aplicar medidas muy estrictas de comparación, halló que el rendimiento del $15 \%$ de los participantes era idéntico al de los nativos.

La existencia de ciertas semejanzas entre el rendimiento de los adultos y el de los nativos también ha sido documentada en el área de la fonética y la fonología. Bongaerts (1999) ha mostrado en varios estudios que algunos hablantes nativos de holandés, a pesar de haber comenzado el estudio de la L2 en la adolescencia, eran capaces de lograr un nivel de pronunciación en inglés y francés prácticamente imposible de distinguir de la pronunciación de los hablantes nativos. En dos de esos estudios, se pidió a un grupo de hablantes nativos de inglés y a dos grupos de holandeses que leyeran en voz alta en inglés un conjunto de frases que contenían sonidos semejantes y distintos a los sonidos del holandés. Una vez aplicada una serie de criterios estrictos de comparación, se descubrió que una proporción significativa de aprendices tardíos tenían un nivel de pronunciación semejante al de los nativos. En un tercer estudio, se analizó la adquisición del francés -una lengua no germánica y no tan frecuente en los medios de comunicación holandeses como lo es el inglés- por parte de aprendices tardíos y, de nuevo, se halló que la pronunciación de un tercio de ellos no se podía diferenciar de la de los nativos.

Existe, además, todo un conjunto de datos sobre la percepción y la producción de sonidos que indican que el problema para los aprendices mayores puede que no sea tanto la pérdida de capacidad lingüística como la falta de acceso a las habilidades correspondientes. Flege (1999) ha mostrado que los adultos sin experiencia previa en estímulos provenientes de la L2 pueden discriminar contrastes fonéticos no-nativos a condición de que dichos contrastes sean suficientemente perceptibles o distintos de las categorías de la L1, de que las pruebas sean lo suficientemente sensibles y no conduzcan a los participantes hacia un procesamiento fonético propio de la L1, y de que se les proporcione suficiente entrenamiento. Otros hallazgos sugieren que la habilidad para pronunciar la L2 a un nivel semejante al de los nativos no se halla totalmente perdida en la edad adulta. Neufeld (1979), por ejemplo, halló que cinco de los siete participantes en su estudio, que tenían un alto nivel de competencia en francés como segunda lengua, fueron evaluados como nativos a partir de su lectura en voz alta de un texto que habían preparado previamente.

La evidencia aportada por este conjunto de estudios ha llevado a autores como MarinovaTodd y otros (2000) a sugerir que la creencia de que los adultos no pueden llegar a dominar la lengua extranjera se debe, entre otras cosas, a la falacia de identificar fracaso con imposibilidad de éxito. Es cierto que la mayor parte de los aprendices adultos terminan con un nivel de dominio de la L2 muy por debajo del de los hablantes nativos. Pero también es cierto que muchos de ellos se enfrascan en la tarea de aprender una nueva lengua sin la suficiente 
motivación, tiempo, energía o apoyo del medio en que se encuentran. La consecuencia, según estos autores, es que tanto los investigadores como los legos se han dejado engañar por el bajo nivel de conocimientos de la mayor parte de los adultos. Y esto, a su vez, ha conducido a desviar la atención de los casos realmente informativos: aquéllos en los que los adultos pueden invertir suficiente tiempo y dedicación a la tarea y se pueden beneficiar de un ambiente que les proporcione el apoyo necesario (véase Manchón y Murphy, 2002).

\subsection{Programas de introducción de las LEs en la escuela}

Por regla general, los resultados de los programas de introducción de lenguas extranjeras en la escuela primaria han mostrado de forma consistente en distintos países europeos que, manteniendo constante el tiempo de contacto con la L2, los niños mayores suelen hacer más progresos que los más pequeños en la adquisición de la morfosintaxis y del léxico y, en algunos casos, también de la fonología (Harley y Wang, 1997). En Suecia, por ejemplo, se enseñó inglés por medio de una metodología audio-visual a unos 1000 niños entre los 8 y los 11 años de edad. Se seleccionó al azar a 355 de estos niños y se evaluó su pronunciación a través de una tarea de imitación y su comprensión auditiva por medio de una tarea basada en la traducción (Ekstrand, 1982). Los resultados de ambas pruebas mostraron que el rendimiento mejoraba de forma lineal con la edad.

En otro programa, que se extendió de 1964 a 1974, se enseñó francés como lengua extranjera a unos 17000 alumnos de 8 años o más que acudían a un conjunto de escuelas seleccionadas en Inglaterra y Gales. Burstall y otros (1974) se encargaron de la evaluación de dicho programa y hallaron que, globalmente, se producía una disminución progresiva de la ventaja que podría haber supuesto el contacto temprano con la lengua extranjera. Una vez que se hubo controlado el tiempo de instrucción, se comparó el rendimiento de un grupo de niños de trece años con el rendimiento de otro grupo de niños de once y se comprobó que la superioridad de los mayores era manifiesta en todas las áreas analizadas. Sin embargo, el resultado más llamativo apareció cuando, a los dieciséis años, se comparó el rendimiento de los que habían tenido una iniciación temprana al francés con el de aquéllos que habían empezado el aprendizaje de esa lengua a la edad "normal" en el sistema educativo británico, es decir, a los once años. No se hallaron diferencias significativas, a excepción de una ligera superioridad del grupo experimental en comprensión auditiva y en actitudes favorables hacia la lengua y la cultura francesas. Hay que señalar que estos resultados tuvieron el efecto secundario de suministrar argumentos a la administración educativa británica para arrinconar la aventura de la introducción temprana de las lenguas en la escuela.

En estudios posteriores llevados a cabo durante los años 90 -período en el que la enseñanza de lenguas extranjeras en primaria llegó a ser una práctica bastante común en Europa- se comparó, a lo largo de los primeros años de la educación secundaria, el rendimiento de alumnos que habían recibido instrucción en primaria con otros alumnos que no la habían recibido (Blondin y otros, 1998). En general, los datos mostraron efectos positivos a favor de los primeros con relación al desarrollo de sus actitudes respecto a la lengua y a la cultura extranjeras, pero muy poco impacto en el desarrollo de las habilidades productivas y de la competencia metalingüística. Si se comparan estos estudios con aquéllos llevados a cabo en contextos naturales de aprendizaje, se observa que en ambos contextos se produce una ventaja inicial en el aprendizaje de la morfosintaxis y del léxico por parte de los adultos o de los 
adolescentes. A largo plazo, sin embargo, esta ventaja inicial es generalmente compensada e incluso superada por los aprendices más jóvenes en contextos naturales, pero no así en contextos formales (Long, 1990; Harley y Wang, 1997).

Con relación a la evaluación del aprendizaje de la L2 en contextos formales es importante destacar dos proyectos de investigación llevados a cabo en el País Vasco y Cataluña, respectivamente. En ambos se analizó el nivel de competencia de un conjunto de alumnos de escuelas públicas que estaban aprendiendo inglés como lengua extranjera, es decir, sin tener la posibilidad de acceder a ella fuera del horario escolar. Los alumnos, además, habían iniciado su aprendizaje a distintas edades (6, 8 y 11 años), si bien habían recibido un número similar de horas de instrucción cuando tuvo lugar la medición de su rendimiento en diversos parámetros (gramática, percepción y producción de sonidos, etc.). En líneas generales, los resultados del proyecto vasco indicaron que, con relación a los aprendices de menos edad, los mayores alcanzaban un dominio general de la L2 con más rapidez (Cenoz, 2003b); obtenían mejores puntuaciones en juicios de gramaticalidad y mostraban un mayor grado de conciencia metalingüística (García Mayo, 2003); tenían mejor percepción de las vocales y consonantes de la L2, su producción presentaba menos acento extranjero y era, por tanto, más inteligible (García Lecumberri y Gallardo, 2003); o escribían textos cuya evaluación global era más alta y cuyos errores mostraban la mayor complejidad de sus enunciados (Lasagabaster y Doiz, 2003). Resultados similares se obtuvieron en el proyecto catalán con relación, entre otros aspectos, a la producción de textos escritos (Torras y Celaya, 2001), la utilización de estrategias de aprendizaje (Victori y Tragant, 2003), o el empleo de ciertas habilidades de producción oral (Muñoz, 2003).

Con relación a este tipo de estudios hay que hacer notar, sin embargo, que, antes de ser considerados como prueba del fracaso de la introducción temprana de las lenguas extranjeras en la escuela, han de tomarse como evaluaciones de distintas versiones de un tipo de enseñanza formal del que no se conoce muy bien el enfoque metodológico utilizado, más allá de unas someras descripciones. Quiere ello decir que la superioridad mostrada por los adolescentes frente a los niños bien podría ser una consecuencia de los instrumentos de evaluación utilizados, quizás más en consonancia con las habilidades a disposición de los primeros que de los segundos. Así Nikolov (2000), al revisar el trabajo de Ekstrand (1982), sugiere que sus resultados bien podrían ser el producto de las técnicas de enseñanza y de evaluación utilizadas, pues tanto las unas como las otras, al favorecer las destrezas de tipo cognitivo/académico, no parecían ser las más adecuadas para los niños pequeños. De forma similar, Cenoz (2003b) no deduce de sus datos que la enseñanza temprana de una lengua extranjera en la escuela sea una alternativa a desechar, pues los niños más pequeños de su estudio, debido a su corta edad, pudieron haber experimentado más dificultades de tipo cognitivo a la hora de realizar las pruebas que los mayores, los cuales pudieron haber desarrollado estrategias más efectivas debido a su experiencia.

Las dificultades metodológicas de los profesores, o sus estrategias de enseñanza, también han sido aducidas como otras posibles explicaciones de los resultados obtenidos. Rixon (1992), por ejemplo, al revisar el estudio de Burtsall y otros (1974), aduce que la puesta en práctica del programa fue bastante desigual entre los diversos profesores seleccionados, debido a sus distintas experiencias de formación y a sus muy diferentes niveles de competencia en francés. Pero, sobre todo, destaca que el principal problema residió en las dificultades que los profesores de secundaria hubieron de afrontar para integrar a los aprendices tempranos en 
clases que también contaban con principiantes absolutos. Todo ello, señala Rixon, puede haber contribuido a la nivelación de los rendimientos de todos los estudiantes, con independencia de la edad a la que comenzaron a aprender la lengua extranjera. Por su parte, Torras y Celaya (2001), al interpretar los resultados de su estudio, señalan que los profesores habían empezado a utilizar una metodología más centrada en la forma lingüística con los alumnos que tenían de 11 a 12 años. Por tanto, la superioridad de los adolescentes pudo deberse no sólo a factores relativos a la edad, sino también a las estrategias de enseñanza empleadas en clase: el frecuente uso de actividades metalingüísticas adecuadas al grado de madurez cognitiva de este tipo de alumnos pudo haber favorecido el desarrollo de su conciencia lingüística. Las autoras, sin embargo, no concluyen que, a partir de estos resultados, haya que posponer la enseñanza de las lenguas extranjeras. Sí señalan, sin embargo, que la principal utilidad de los datos aportados quizá resida en que pueden ayudar a los profesores de primaria a formarse expectativas válidas sobre el rendimiento de los alumnos a distintas edades.

En cualquier caso, no hay que olvidar que en contextos formales de aprendizaje no siempre es posible que los niños pequeños obtengan mejores resultados a medio y largo plazo que los adultos o que otros niños de más edad, simplemente porque el medio les impide obtener la cantidad de input necesaria para ello. A este respecto, señala Singleton (1995) que el número de horas de contacto con la segunda lengua durante un año en un contexto natural suele ser superior al número de horas habitualmente dedicadas a la lengua extranjera en toda la escolarización (véase Cenoz y Perales, 2000, para una revisión de estudios sobre estos dos contextos).

\section{Conclusiones}

Revisar la investigación sobre el factor edad supone constatar la enorme cantidad de factores que interactúan a la hora de avalar o rechazar las hipótesis propuestas, así como percatarse de la complejidad de los problemas metodológicos implicados en la obtención de los datos. El resultado es la gran contraposición de hallazgos y de interpretaciones que se encuentran en la literatura. A pesar de ello, quizá siga teniendo validez, al menos en contextos naturales, la conclusión de Singleton (1989: 137): "Con relación a la hipótesis de que aquellos que comienzan a aprender una segunda lengua en la niñez alcanzan a largo plazo un nivel más alto de dominio de la L2 que aquellos que comienzan más tarde, se puede decir que hay evidencia que apoya esa asunción y que no hay contraevidencia”. Por lo que respecta a los contextos formales, que son los que realmente a nosotros nos interesan, los estudios presentados más arriba indican que se ha hallado poca evidencia que avale tal conclusión: en estos contextos, el entusiasmo rara vez es acompañado por los hechos. De cualquier modo, hay razones para suponer que un inicio temprano en las lenguas extranjeras puede contribuir, al menos, a incrementar la motivación de los aprendices, lo que en el futuro les puede ayudar a aumentar su nivel de competencia y a desarrollar sus actitudes de aceptación de otros pueblos, países y culturas. Como para la potenciación de esas capacidades, la exposición, la práctica y la interacción en la L2 son factores clave, podríamos concluir con Hatch (1983) que "cuanto antes sea posible, mejor".

Sin embargo, dada la contraposición de hallazgos y de puntos de vista sobre el tema, "tanto los padres como la administración educativa deberían obrar con cautela a la hora de 
traducir lo que puedan leer sobre la hipótesis del periodo crítico a su práctica personal o a la política del ministerio" (Scovel, 2000: 220). En efecto, uno de los errores que se pueden cometer a la hora de introducir la enseñanza de las lenguas extranjeras en los estadios iniciales de la enseñanza es justificar tal decisión apelando a las ventajas derivadas de la introducción temprana en un contexto natural. Ante ello, se trata de ser realistas sobre lo que se puede esperar de los niños pequeños en contextos de aprendizaje formal, pues, como se ha mostrado más arriba, en estos contextos las cotas de aprendizaje de la L2 suelen ser muy modestas (Singleton, 1995). Otro error consistiría en intentar compensar los costes del adelanto rebajando la intensidad del contacto con la L2. Sin embargo, en este caso habría que tener en cuenta que, incluso tratándose de programas razonablemente intensivos, suele ocurrir que en infantil sólo se puede cubrir la mitad del contenido que se cubriría en primaria, y que en este nivel, a su vez, se suele progresar de forma más lenta que en secundaria (Marinova-Todd, 2000). Finalmente, habría que sopesar las repercusiones de la introducción temprana de la L2 sobre los otros componentes del currículo, ya que hasta el momento no hay suficientes estudios en los que se demuestre que merece más la pena enseñar lenguas extranjeras que invertir más tiempo en la enseñanza de las matemáticas, las ciencias, el arte, e incluso la lengua materna. En este sentido, hay que señalar que, en una de las pocas revisiones sobre el tema, Collier (1992), después de analizar un conjunto de estudios sobre niños bilingües en los estadios iniciales de la escolaridad, concluyó que la instrucción en lengua materna es más importante que la instrucción en segundas lenguas para la consecución de determinados objetivos académicos.

Teniendo todo ello en cuenta, quizá una de las recomendaciones más sensatas sea que la introducción de las lenguas extranjeras en la escuela se debería iniciar tan pronto como las condiciones materiales, sociales y educativas sean tales que favorezcan la posibilidad de una experiencia de aprendizaje positiva. Tratando de afinar lo más posible, entre estas condiciones se podrían citar las siguientes (Cenoz, 2003a): (i) comprender que se trata de un proyecto a largo plazo que implica la adaptación del currículum y de los materiales a los alumnos a los que va dirigido; (ii) establecer objetivos bien definidos y realistas, teniendo en cuenta el entorno, el centro y las horas de instrucción disponibles; (iii) aplicar una metodología efectiva y adecuada a la edad de los alumnos; (iv) coordinar la interdependencia entre las lenguas a fin de obtener beneficios a nivel cognitivo y metalingüístico; (v) conseguir el apoyo de los miembros de la comunidad escolar para llevar a cabo el proyecto; y (vi) garantizar la formación lingüística y metodológica del profesorado.

En resumen, la cuestión de la enseñanza de la lenguas extranjeras en la escuela no tendría que ver tanto con el cuándo cuanto con el cómo hacerlo y en qué contexto. Se trata de un tema que, para decirlo con Vez y otros (2002: 76), tiene que ver no sólo "con aspectos neurofisiológicos, sino también con la naturaleza de ciertos factores sociales, económicos, políticos y psicológicos que inciden de lleno en esta cuestión de la planificación educativa". 


\section{RefERENCIAS}

Bialystok, E. y Hakuta, K. (1999). "Confounded age: Linguistic and cognitive factors in age differences for second language acquisition", en D. Birdsong, (ed.), Second language acquisition and the critical period hypothesis. Mahwah, NJ: Lawrence Erlbaum, Associates, 161-181.

Birdsong, D. (1992). "Ultimate attainment in second language acquisition", en Language, 68, 4: 706-755.

Birdsong, D. (1999). Second Language Acquisition and the critical period hypothesis. Mahwah, NJ: Lawrence Erlbaum Associates.

Blondin, C., Candelier, M., Edelenbos, P., Johnstone, R., Kubanek-German, A. y Taeschner, T. (eds.) (1998). Foreign languages in primary and pre-school education. London: CILT.

Bongaerts, T. (1999). "Ultimate attainment in L2 pronunciation: The case of very advanced late L2 learners", en D. Birdsong (ed.), Second language acquisition and the critical period hypothesis. Mahwah, NJ: Lawrence Erlbaum, Associates, 133-159.

Burstall, C., Jamieson, M. Cohen, S. y Hargreaves, M. (1974). Primary French in the balance. Windsor: NFER Publishing Company.

Cenoz, J. (2003a). "Learning English in kindergarten: cognitive, linguistic and affective effects University of the Basque Country", en EDULING, Instituto de Ciencias de la Educación de la Universidad de Barcelona. Marzo.

Cenoz, J. (2003b). "The influence of age on the acquisition of English: General proficiency. Attitudes and code-mixing”, en M.P. García Mayo y M.L. García Lecumberri (eds.), Age and the acquisition of English as a foreign language. Clevedon: Multilingual Matters, 7793.

Cenoz, J. y Perales, J. (2000). "Las variables contextuales y el efecto de la instrucción en la adquisición de segundas lenguas", en C. Muñoz (ed.), Segundas lenguas. Adquisición en el aula. Barcelona: Ariel, 109-125.

Collier, V.P. (1992). "A synthesis of studies examining long-term language minority student data on academic achievement", en Bilingual Education Research Journal, 16, 1 y 2: 187-221.

Cook, V. (1992). "Evidence for multicompetence", en Language Learning, 42, 4: 557-591.

Cranshaw, A. (1997). A study of Anglophone native and near-native linguistic and metalinguistic performance. Tesis doctoral no publicada, Université de Montréal.

De Keyser, R. (2000). "The robustness of critical period effects in second language acquisition", en Studies in second language acquisition, 22, 4: 499-533.

Ekstrand, L. (1982). "English without a book revisited: The effect of age on second language acquisition in a formal setting", en S. Krashen, M. Long y R. Scarcella (eds.), Child/adult differences in second language acquisition. Rowley, MA: Newbury House, 123-135.

Flege, J.E. (1999). "Age of learning and L2 speech", en D. Birdsong (ed.), Second language acquisition and the critical period hypothesis. Mahwah, NJ: Lawrence Erlbaum, Associates, 101-131.

Flege, J.E., Munro, M.J. y Mackay, I. (1995). "Factors affecting the strength of perceived foreign accent in a second language", en Journal of the Acoustical Society of America, 97, 5: 31253134.

Flege, J.E., Yeni-Komshian, S. y Liu, S. (1999). "Age constraints on second-language acquisition”, en Journal of Memory and Language, 41, 1: 78-104.

García Lecumberri, M.L. y Gallardo F. (2003). "English FL sounds in school learners of different ages", en M.P. García Mayo y M.L. García Lecumberri (eds.) Age and the acquisition of English as a foreign language. Clevedon: Multilingual Matters, 115-135. 
García Mayo, P. (2003). "Age, length of exposure and grammaticality judgements in the acquisition of English as a foreign language", en M.P. García Mayo y M.L. García Lecumberri (eds.) Age and the acquisition of English as a foreign language. Clevedon: Multilingual Matters, 94-114.

Gleitman, L y Newport, E. (1995). "The invention of language by children: environmental and biological influences on the acquisition of language", en L. Gleitman y M. Liberman (eds.), Language: An invitation to cognitive science. Vol. 1. Cambridge, MA: MIT Press, 1-24.

Harley, B. y Wang, W. (1997). "The critical period hypothesis: Where are we now?", en A.M.B. de Groot y J.F. Kroll (eds.), Tutorials in bilingualism: Psycholinguistic perspectives. Mahwah, NJ: Erlbaum, 19-51.

Hatch, E. (1983). Psycholinguistics: A second language perspective. Rowley, Mass.: Newbury House.

Hyltestam, K. (1992). "Non-native features of near native speakers: On the ultimate attainment of childhood L2 learners", en R. J. Harris (ed.), Cognitive processing in bilinguals. Mawah, NJ: Erlbaum, 19-51.

Hyltestam, K. y Abrahamsson, (2003). "Maturational constraints in SLA", en C.J. Doughty y M.H. Long (eds.), The handbook of second Language acquisition. Oxford: Blackwell Publishing, 539-588.

Johnson, J.S. y Newport, E.L. (1989). "Critical period effects in second language learning: The influence of maturational state on the acquisition of English as a second language", en Cognitive Psychology, 21, 1: 60-99.

Johnson, J.S. (1992). "Critical period effects in second language acquisition: The effect of written versus auditory materials on the assessment of grammatical competence", en Language Learning, 42, 2: 217-248.

Kim, K.H.S., Relkin, N.R., Kyoung-Min, L., y Hirsch, J. (1997). "Distinct cortical areas associated with native and second languages", en Nature, July, 388: 171-174.

Lasagabaster, D. y Doiz, A. (2003). "Maturational constraints on foreign-language written production", en M.P. García Mayo y M.L. García Lecumberri (eds.) Age and the acquisition of English as a foreign language. Clevedon: Multilingual Matters, 136-160.

Long, M. (1990). "Maturational constraints on language development", en Studies in Second Language Acquisition, 12, 3: 251-285.

Mägiste, E. (1987). "Further evidence for the optimal age hypothesis in second language learning", en J. Lantolf y A. Labarca (eds.) Language Learning: Focus on the classroom. Norwood, NJ Ablex, 51-58.

Manchón Ruiz, R. M. y Murphy, L. (2002). An introduction to second language acquisition research. Murcia: ICE Universidad de Murcia.

Marinova-Todd, S. H., Bradford Marshall, D. y Snow, C. (2000). "Three misconceptions about age and second language learning", en TESOL Quarterly, 34, 1: 9-34.

Morford, J.P. y Mayberry, R.I. (2000). "A re-examination of "early exposure" and its implications for language acquisition by eye”, en C. Chamberlain, J.P. Morford y R.I. Mayberry (eds.), Language acquisition by eye. Mahwah, NJ: Lawrence Erlbaum Associates, 111-127.

Muñoz, C. (2003). "Variation in oral skills development and age of onset", en M.P. García Mayo y M.L. García Lecumberri (eds.) Age and the acquisition of English as a foreign language. Clevedon: Multilingual Matters, 161-181.

Neufeld, G. (1979). "Towards a theory of language learning ability", en Language Learning, 29, 2: $227-241$. 
Nikolov, M. (2000). "Issues in research into early foreign language programs", en J. Moon y M. Nikolov (eds.) Research into English to young learners. University Press Pécs. Pécs, 2148.

Patkowski, M.S. (1980). "The sensitive period for the acquisition of syntax in a second language", en Language Learning, 30, 2: 449-472.

Piske, T., Mackay, I.R.A. and Flege, J.E. (2001). "Factors affecting degree of foreign accent: a review", en Journal of Phonetics, 29, 2: 191-215.

Rixon, S. (1992). "English and other languages for younger children: Practice and theory in a rapidly changing world", en Language Teaching, 25, 2: 73-93.

Ruben, R.J. (1997). "A time frame of critical/sensitive periods of language development", en Acta Otolaryngologica, 117, 2: 202-205.

Schachter, J. (1996). "Maturation and the issue of universal grammar in second language acquisition", en W. C. Ritchie y T. K. Bhatia (eds.), Handbook of Second Language Acquisition. San Diego: Academic Press, 159-193.

Scovel, T. (2000). "A critical review of the critical period research", en Annual Review of Applied Linguistics, 20: 213-233.

Selinker, L. (1972). "Interlanguage". International Review of Applied Linguistics. 10, 2: 209-231. Singleton, D. (1989). Language acquisition: The age factor. Clevedon, Avon: Multilingual Matters. Singleton, D. (1995). "Second language in primary school: The age dimension", en TEANGA: The Irish Yearbook of Applied Linguistics, 15: 155-166.

Singleton, D. (2001). "Age and second language acquisition", en Annual Review of Applied Linguistics, 21: 77-89.

Singleton, D. (2003). "Critical period or general age factors?", en M.P. García Mayo and M.L. García Lecumberri (eds.), Age and the acquisition of English as a foreign language. Clevedon: Multilingual Matters, 3-22.

Skehan, P. (1998). A cognitive approach to language learning. Oxford: OUP.

Thompson, E. (1991). "Foreign languages revisited: The English pronunciation of Russian immigrants", en Language Learning, 41, 2: 177-204.

Torras, M.R. y Celaya, M.L. (2001). "Age related difference in the development of written production", en International Journal of English Studies, 1, 2: 103-126.

Torras, M.R., Tragant, E., y García, M. P. (1997) "Croyances populaires sur l'apprentissage précoce d'une langue étrangère", en AILE, 10: 127-158.

Vez, J. M., Guillén, C. y Alario, C. (2002). 'Didáctica de la lengua extranjera en educación infantil y primaria. Madrid: Síntesis.

Victori, M. y Tragant, E. (2003). "Learner strategies: A cross-sectional and longitudinal study of primary and high-school EFL learners", en M.P. García Mayo and M.L. García Lecumberri (eds.), Age and the acquisition of English as a foreign language. Clevedon: Multilingual Matters, 182-209.

Weber-Fox, C. y Neville, H. (1999). "Functional neural systems are differentially affected by delays in second language immersion: ERP and behavioral evidence in bilinguals", en D. Birdsong (ed.), Second language acquisition and the critical period hypothesis. Mahwah, NJ: Lawrence Erlbaum, Associates, 23-38.

White, L. y Genesee, F. (1996). "How native is near native? The issue of ultimate attainment in adult second language acquisition", en Second Language Research, 12, 3: 238-265. 